\title{
TWO ENUMERATIVE PROOFS OF AN IDENTITY OF JACOBI
}

\author{
by C. SUDLER, JR. \\ (Received 22nd January 1965)
}

\section{Introduction}

In (7), Wright gives an enumerative proof of an identity algebraically equivalent to that of Jacobi, namely

$$
\prod_{n}\left(1+x^{2 n-1} z\right)\left(1+x^{2 n-1} z^{-1}\right)=\prod_{n}\left(1-x^{2 n}\right)^{-1} \sum_{r} x^{r^{2}} z^{r}
$$

Here, and in the sequel, products run from 1 to $\infty$ and sums from $-\infty$ to $\infty$ unless otherwise indicated. We give here a simplified version of his argument by working directly with (1), the substitution leading to equation (3) of his paper being omitted. We then supply an alternative proof of (1) by means of a generalisation of the Durfee square concept utilising the rectangle of dimensions $v$ by $v+r$ for fixed $r$ and maximal $v$ contained in the Ferrers graph of a partition.

After receiving a pre-publication copy of (7), I discovered that Sylvester ((5), pp. 34-36) had already given a proof of (1) of the required type. However, perhaps because of his somewhat verbose and unclear style, his work on this topic has apparently been almost completely ignored in recent times except by MacMahon, who gave ((4), $\S 323)$ a generalisation of Sylvester's idea. Thus all the familiar proofs have involved analysis ((3), pp. 282-283) or at least rather tricky algebraic arguments involving polynomials and series ((2), pp. 282-283). Furthermore, the two proofs given below are considerably more transparent than Sylvester's, though in a sense variants of it, and provide a clear insight into the nature of (1).

It seems desirable, moreover, to provide a complete enumerative proof of Jacobi's identity in its original form, namely

$$
\prod_{n}\left(1-x^{2 n}\right)\left(1+x^{2 n-1} z\right)\left(1+x^{2 n-1} z^{-1}\right)=\sum_{r} x^{r^{2}} z^{r}
$$

Given (1), this problem reduces to the corresponding one for

$$
\prod_{n}\left(1-x^{n}\right) \prod_{v}\left(1-x^{v}\right)^{-1}=1,
$$

which has been solved by Vahlen ((1), p. 146). However, we can supply a more direct proof by establishing a simple one-to-one correspondence.

\section{Notation and conventions}

Unless otherwise indicated, small Roman and Greek letters will denote 
positive integers and the summands of finite sums such as $\sum_{1}^{u} c_{t}=\sum_{t=1}^{u} c_{t}$ will be arranged in non-decreasing order. In sums or products, the index will always be a subscript or an exponent and will be omitted (like $t$ above) when there is no risk of ambiguity. Empty sums or products are to be interpreted as 0 or 1 respectively, and all correspondences are one-to-one.

In the following we shall be concerned with certain representations of an integer $h \geqq 0$ as a sum and we make the convention that 0 has one representation or partition of the type being considered without explicit mention. We ignore the conditions of validity of formal operations with series and products, which are well-known and, moreover, essentially irrelevant in the present context (see (3), p. 276).

The reader is referred to (3), Chapter 19 for concepts not explained in the text. Of the other references, we shall only assume knowledge of (7) in the main body of the text.

\section{Proof of (2) based on Wright's method}

We write the right member of (2) as $\sum_{m=0}^{\infty} \sum_{r=-\infty}^{\infty} C_{m r} x^{m} z^{r}$ and prove that

$$
C_{m r}=\left\{\begin{array}{l}
1, m=r^{2} \\
0, m \neq r^{2} .
\end{array}\right.
$$

Consider for fixed $r$ any representation $R$ of $m$ of the form

$$
m=\sum_{1}^{v+r}\left(2 a_{t}-1\right)+\sum_{1}^{v}\left(2 b_{t}-1\right)+\sum_{1}^{u} 2 c_{t} ; v \geqq 0, u \geqq 0,
$$

where the summands in each sum are distinct. Now on expanding the product in (2) formally, we find without difficulty that

$$
C_{m r}=\sum_{R}(-1)^{u}
$$

where the sum is extended over all representations of $m$ in the form (5). Then, by (5), $C_{m,-r}=C_{m r}$, so that we may take $r \geqq 0$ in the sequel. Next, (5) is equivalent to

$$
(m+r) / 2=\alpha+\gamma ; \alpha=\sum_{1}^{v+r} a_{t}+\sum_{1}^{v}\left(b_{t}-1\right), \gamma=\sum_{1}^{u} c_{t} .
$$

Now if $m<r^{2},(7)$ is clearly insoluble since $a_{t} \geqq t$, whereas if $m=r^{2}, v=u=0$ and $a_{t}=t$ only. Hence by (6),

$$
C_{m r}=\left\{\begin{array}{l}
0, m<r^{2} \\
1, m=r^{2} .
\end{array}\right.
$$

Assume henceforth that $m>r^{2}$ and write $k=x-r(r+1) / 2$. Then there is a correspondence, given by the second figure in (7), between the representations of $\alpha$ given by (7) and the unrestricted partitions of $k$. (Our notations and conventions agree with equation (6) of (7) except that his $n$ is our $\alpha$ ). 
Let $\sum_{1}^{h} e_{g}$ be an unrestricted partition of $k$. Then by (7)

$$
\mu=\gamma+k=\left(m-r^{2}\right) / 2=\sum_{1}^{\mu} c_{t}+\sum_{1}^{h} e_{g}, \quad c_{t}<c_{t+1} \quad(t \geqq 1)
$$

in which $\mu>0$ since $m>r^{2}$ by hypothesis. Consider the composite partition of $\mu$ given by (9) which we denote by $B=B_{\mu}$. Thus by (6),

$$
C_{m \mathrm{r}}=\sum_{B}(-1)^{u} \text {, }
$$

which is, of course, the coefficient of $x^{\mu}$ in the expansion of (3). We now write

$$
\sum_{i}^{h} e_{g}=\sum_{a}^{b} i v_{i}
$$

where $v_{i} \geqq 0$ is the multiplicity of $i$ among the $e_{g}$ but $v_{a}>0, v_{b}>0$. Then $B_{\mu}$ corresponds to the partition

$$
\beta=\beta_{\mu}=\left(c_{1}, \ldots, c_{u} \mid a v_{a}, \ldots, b v_{b}\right) ; c_{1}<\ldots<c_{u} .
$$

We now establish a correspondence between the $\beta$ with $u$ even and those with $u$ odd. Then on replacing $B$ by $\beta$ in (10), we shall have

$$
C_{m r}=0, m>r^{2} \text {, }
$$

and the proof of (4) is complete by (8). We distinguish two cases in (11).

(i) $c_{1} \leqq a$ or the $v_{i}$ absent. Then we take $\beta$ into

$$
\beta^{\prime}=\left\{\begin{array}{l}
\left(c_{2}, \ldots, c_{u} \mid c_{1}, a v_{a}, \ldots, \ldots, b v_{b}\right), c_{1}<a \\
\left(c_{2}, \ldots, c_{u} \mid a\left(v_{a}+1\right), \ldots, b v_{b}\right), c_{1}=a .
\end{array}\right.
$$

(ii) $c_{1}>a$ or the $c_{t}$ absent. Then we take $\beta$ into

$$
\beta^{\prime}=\left(a, c_{1}, \ldots, c_{u} \mid a\left(v_{a}-1\right), \ldots, b v_{b}\right) .
$$

It is clear that if $\beta$ satisfies (i), $\beta^{\prime}$ satisfies (ii) and conversely, so that we have the required correspondence and (12) is proved.

We next consider a result of Vahlen's (6), a direct proof of which is an immediate corollary of the preceding analysis. Let $S$ be a representation of $l \geqq 0$ in the form

$$
l=\sum_{1}^{v+r}\left(3 a_{t}-1\right)+\sum_{1}^{0}\left(3 b_{t}-2\right)+\sum_{1}^{u} 3 c_{t}
$$

in which the conditions of (5) hold, and put $A_{l r}=(-1)^{r} \sum_{S}(-1)^{u}$, which is in effect the function evaluated by Vahlen. We then have, by (13),

$$
(l+r) / 3=\sum_{1}^{v+r} a_{t}+\sum_{1}^{v}\left(b_{t}-1\right)+\sum_{1}^{u} c_{t}
$$

Now the right members of (14) and (7) are identical, so that

$$
A_{i r}=(-1)^{r} C_{m r},(l+r) / 3=(m+r) / 2 \text {. }
$$


Then $l=(3 m+r) / 2$, and by (4), after putting $-r$ for $r$,

$$
A_{l r}=\left\{\begin{array}{r}
(-1)^{r}, l=\left(3 r^{2}-r\right) / 2 \\
0, l \neq\left(3 r^{2}-r\right) / 2
\end{array}\right.
$$

which is Vahlen's result. Our variant of Wright's method is considerably simpler than Vahlen's original proof.

To conclude this section, we note that a direct proof of Jacobi's identity for $\Pi\left(1-x^{n}\right)^{3}((3)$, Theorem 357) can also be obtained using the method above, by working with $\Pi\left(1-x^{n}\right)\left(1-x^{n} z\right)\left(1-x^{n} z^{-1}\right)((3), 19.9 .4$, with $z=-\zeta)$. However, the details are a little tedious and not very interesting combinatorially.

\section{Alternative proof of (1)}

Put $\Pi\left(1+x^{2 n-1} z\right)\left(1+x^{2 n-1} z^{-1}\right)=\Sigma z^{r} f_{r}(x)$. Now $f_{r}(x)$ enumerates representations of $m \geqq 0$ in the form

$$
m=\sum_{1}^{v+r}\left(2 a_{t}-1\right)+\sum_{1}^{v}\left(2 b_{t}-1\right), v \geqq 0,
$$

where the conditions of (5) hold. As before, we may take $r \geqq 0$ and (15) is then equivalent to

$$
\begin{aligned}
\left(m-\left\{(v+r)^{2}+v^{2}\right\}\right) / 2 & =\left(m-r^{2}\right) / 2-v(v+r) \\
& =\sum_{1}^{v+r}\left(a_{t}-t\right)+\sum_{1}^{v}\left(b_{t}-t\right),
\end{aligned}
$$

where the last two sums are unrestricted partitions (with non-negative summands). By (16) we have $x^{-r^{2}} f_{r}(x)=g_{r}\left(x^{2}\right)$ where

$$
g_{r}(y)=\sum_{0}^{\infty} y^{v(v+r)} \prod_{1}^{v+r}\left(1-y^{s}\right)^{-1} \prod_{1}^{v}\left(1-y^{s}\right)^{-1}=\prod_{1}^{\infty}\left(1-y^{n}\right)^{-1},
$$

in which the last equality arises by comparison with (1). For $r=0$ this is familiar ((3), Theorem 351), and we prove (17) by generalising the pictorial argument used there.

We denote by $P$ any partition of $h$ such that $h=d_{1}+\ldots+d_{\rho}$, with $d_{1} \geqq \ldots \geqq d_{\rho}$. Let $F$ be the Ferrers graph of $P$ and identify the nodes of $F$ with a finite set of lattice points $(p, q)$ such that $1 \leqq p, 1 \leqq q$ and that $(p, 1)$ is a node of $F$ for $1 \leqq p \leqq d_{1}$. Consider for fixed $r \geqq 0$ the nodes $(r+\lambda, \lambda)$, which occur if $d_{1} \geqq r+1$, and let $j$ be the maximum value of $\lambda$. Then the nodes $(p, q)$ with $p \leqq r+j, q \leqq j$ form a unique rectangle $D_{r}$ " inscribed" in $F$ such that the base and altitude differ by $r$. If $D_{r}$ does not occur, we say that $j=0$. For $j>0$, every partition $P$ is uniquely dissected into $D_{r}$ and two " tails" representing partitions into at most $j+r$ and at most $j$ parts resp. $P$ is then enumerated by that term of the sum in (17) for which $v=j$, which is also true for $j=0$ since we are then considering partitions with at most $r$ parts. 
In response to my inquiry about its occurrence in the literature, Dr Wright pointed out that (17) can be derived from the main formula of ((4), § 263) as follows. We interchange MacMahon's $a$ and $x$, put $x^{r}$ in place of $a$, and multiply through by $\prod_{1}^{r}\left(1-x^{5}\right)^{-1}$.

\section{Acknowledgments}

Vahlen's result in (6) was quoted in an earlier version of (7) made available to me, which drew my attention to it. I am indebted to Dr Wright for the pre-publication copy of his paper and for discussion and correspondence regarding the contents of this note.

\section{REFERENCES}

(1) L. E. Dickson, History of the Theory of Numbers, II, New York (Chelsea, 1952).

(2) G. H. HARDY and E. M. Wright, An Introduction to the Theory of Numbers (3rd edition Oxford, 1954).

(3) G. H. HARDY and E. M. WRIGHT, An Introduction to the Theory of Numbers (4th edition Oxford, 1960).

(4) P. A. MacMahon, Combinatory Analysis, II (Cambridge, 1916).

(5) J. J. Sylvester, A constructive theory of partitions, Collected Papers, IV (Cambridge, 1912), 1-83.

(6) K. T. VAhLEN, Beiträge zu einer additiven Zahlentheorie, J. für Math. 112 (1893), 1-36.

(7) E. M. Wright, An enumerative proof of an identity of Jacobi, J. London Math. Soc. 40 (1965), 55-57.

LOS ANGELES

CALIFornia 